\title{
RAPID AUTOMATIZED NAMING IN EARLY LITERACY
}

\author{
Roberta Ceccato \\ Associate professor at International University of Valencia (VIU) roberta.ceccato@campusviu.es \\ $M^{a}$ Dolores Gil Llario \\ Department of Evolutionary Psychology and Education \\ University of Valencia. General Studi \\ dolores.gil@uv.es \\ Mà Isabel Marí-Sanmillán \\ Department of Education Sciences \\ CEU Cardenal Herrera University. Castellón \\ maria.mari1@uchceu.es
}

Fecha de Recepción: 9 Abril 2019

Fecha de Admisión: 30 Abril 2019

\begin{abstract}
Although the relation between Rapid Automatized Naming (RAN) and Reading is clear, there is no a standardized test to measure the speed of naming in early literacy. The aim is to explore and confirm the factorial structure of the RAN test developed by Wolf and Denkla (2003) in children. RAN test was administred to 442 children aged 4-5 years. Results highlighted two factors: a "nonalphanumeric components" factor which includes "RAN colors" and "RAN images" and an "alphanumeric components" factor which collects "RAN letters" and "RAN numbers". Moreover, results highlighted a gradual shift of weight that each component of the test has in the two analyzed years and a consequent reduction in the discrimination ability of the test. In conclusion, this study not only allows to analyze the structure of the instrument "RAN", but also describes how the speed of naming works over the early literacy.
\end{abstract}

Keywords: RAN; early literacy; structural model

Cognitive aspects related to reading learning process and its development are an important and current research topic because they help us understand the nature of neural processes underlying reading. A basic cognitive skill that is often associated with reading ability is the rapid and automatic recognition of visual items (Kirby, Roth, Desrochers \& Lai, 2008). This rapid decoding (Rapid Automatized Naming, from now on RAN) refers to the time it takes for a child to quickly and accurately name a number of familiar visual stimuli (usually letters, digits, objects and colors). 
Norton and Wolf (2011) recently argued that the RAN is "a microcosm or mini-circuit of the later-developing reading circuitry" appealing to the fact that both systems involve closely related cognitive processes. Indeed, both RAN tasks and the reading process involve: attention to the stimulus; visual processes responsible for the initial detection of the stimuli's characteristics; decoding and grapheme-phoneme conversion; integration of visual information with spelling and phonological representations stored in memory; lexical processes such as access and retrieval of phonological codes; and organization of the articulation production (Araújo, Petersson, Reis \& Faísca, 2014).

A substantial body of research identifies RAN as one of the best (and perhaps one of the most universal, longitudinal and current) predictors of reading ability and also of the detection of a possible deficit in its development (Araújo, Inácio, Francisco, Faísca, Petersson \& Reis, 2011; Kirby, Parrila \& Pfeiffer, 2003; Kirby et al., 2008; Vaessen, Bertrand, Tóth, Csépe, Faísca, Reis \& Blomert, 2010; Wolf, Bowers \& Biddle, 2000; Wolf, O’Rourke, Gidney, Lovett, Cirino \& Morris, 2002).

There are many theories that attempt to analyze and define the relationship between RAN and reading and, currently, there is no common consensus on which mechanisms are responsible for this relationship. While some authors suggest that the RAN primarily reflects the access and recovery in long-term memory of phonological codes (Chiappe, Stringer, Siegel \& Stanovich, 2002; Pennington, Cardoso-Martins, Green \& Lefly, 2001; Schatschneider, Carlson, Francis, Foorman \& Fletcher, 2002), others support the hypothesis that the RAN is a reader "non-phonological" independent process (Wolf \& Bowers, 1999). Supporting this hypothesis, Bowers et al. proposed the existence of an orthographic (and not phonemic) base in the relationship between RAN and reading processes (Bowers \& Newby-Clark, 2002; Bowers \& Wolf, 1993; Wolf at al., 2000). This hypothesis has received support in recent years by different authors and empirical confirmation (Araújo et al., 2011; Georgiou, Parrila, Kirby \& Stephens, 2008; Roman, Kirby, paririla, Wade-Woolley \& Deacon, 2009), although it has been strongly criticized by other researchers (Cutting \& Denckla, 2001; Moll, Fussenegger, Willburger \& Landerl, 2009; Papadopoulos, Georgiou \& Kendeou, 2009).

Furthermore, this disagreement refers not only to the origin of the relationship between RAN and reading, but also to the intensity of the relationship. Some studies report that there is a close correlation between the measurements of the speed in naming and reading performance (Babayig it \& Stainthorp, 2010), others emphasize a weak relationship (Cunningham, 2006) and finally some highlights an almost negligible relationship (Scaborough, 1998).

One of the main causes of absence in the literature of a common consensus on the relationship between RAN and reading skills is the variety of methods of measuring the speed of naming used by researchers. These differences are, for example, in the type of stimuli used. Some studies emphasize that scores on the RAN in "non-alphabetic" stimuli (such as objects and colors) represent, before the child began his schooling education, a good predictor of later development of reading (Kirby at al., 2003; Landerl \& Wimmer, 2008). However, as De Jong (2011) emphasizes, when the reading-writing development of children and their schooling already begun, the "alphanumeric" RAN stimuli (letters and numbers) acquire greater importance and relationship with the reading ability than "non-alphanumeric" items of colors and objects.

Likewise, there are other differences in the methods of measuring the speed of naming that may have affected the considerations about his relationship with reading processes. Some studies, for example, have changed the length of the tasks of the RAN, diverging of the 50 items for each type of stimulus ( 50 colors, 50 objects, 50 letters and 50 numbers divided into 4 different sheets) raised in the original model. In addition, some studies also increases the variety of stimuli changing deeply the original paradigm in which five different items are repeated to form the 50 stimuli sheet 6 in Georgiou et al., 2008; 20 in Cobbold, Passenger \& Terrel, 2003 and 25 in Clarke, Hulme \& Snowling, 2005). 
In 2011 Norton \& Wolf stressed that although small differences in the length of the tasks of the RAN may not necessarily have a strong impact on the measurement provided if the familiarity of the stimuli is ensured, changes in the variety of these stimuli can directly affect the performance of children in these tasks. Indeed, according to these authors, increasing the number of types of stimuli that the child has to recognize and recover in the long-term memory, the degree of difficulty of the task will also increase.

From these observations, it is clear the need to develop a consensual and standardized test to measure in a common and equal way the naming speed, allowing thus to analyze more reliably not only the performance, but also its relationship with the reading development. Thus, the aim of this study is to explore and confirm the factorial structure of the RAN test developed by Wolf and Denkla in 2003 with the purpose of making available to different researchers a reliable and standardized test for measuring naming speed.

\section{METHOD}

\section{Participants}

The study involved a total of 219 children aged 4 years and 223 children aged 5 years. 110 of the 219 four years children were boys $(50.4 \%)$ and 109 were girls $(49.6 \%)$ and were taking $2^{\text {nd }}$ grade of Kindergarten; 112 of the 223 five years students, were boys $(50.4 \%)$ and 111 were girls $(49.6 \%)$ and were taking 3rd grade of kindergarten. All students were in the same school from the age of 3 .

To explore and confirm the factorial structure of the test, we proceeded first to randomly divide the four years participants into two subsamples. The first sub-sample consisted of 100 subjects ( $49 \%$ girls and $51 \%$ boys) and was used for carrying out the exploratory factor analysis (EFA). The second subsample with 119 participants ( $50.4 \%$ boys and $49.6 \%$ girls) was used to perform the Confirmatory Factor Analysis (CFA). The same procedure was applied to the participants of five years, obtaining two subsamples: one of 100 subjects (50\% girls and $50 \%$ boys) for carrying out the EFA and another made up of 123 participants (49.6\% girls and $50.4 \%$ boys) to performing CFA.

\section{Instrument}

The rapid Automatized Naming Test (RAN; Wolf y Denkla, 2003). The task consists in naming 200 stimuli grouped into four subtests (numbers, letters, colors and familiar images), as quickly as possible. Stimuli are presented in $21 \times 30 \mathrm{~cm}$ different cardboards organized into five rows of 10 stimuli each. They are organized randomly but always 50 of all of them. The evaluator has to list the time it takes for the student to name all the stimuli of each card and the number of errors committed by naming. An efficiency ratio of each subtest is obtained according to the procedure described by Compton (2003) of converting scores into numbers/second, letters/second, colors/second and images/second (as the original instrument proposal indicates).

\section{RESULTS}

First of all, a statistical analysis of the items making up the test in both samples was conducted. As shown in Table 1, the mean scores obtained in the various components of the RAN by children 4 years range between 1,873 and 2,739 and placed on five years between 1,120 and 1,503. Likewise, it's possible to notice that the group of participants in this study showed a positive skewed distribution, except for the components of RAN numbers and RAN letters in children 4 years who had a negatively skewed distribution. Furthermore, while the group of participants of five years presents a platykurtic distribution in all components, for children of 4 years old this happens only in 
the components of RAN numbers and RAN colors, being RAN letters and RAN images characterized by a leptokurtic distribution. Thus, we can conclude that the group of participants in this study presents a non-normal sampling distribution.

Table 1. Psychometric properties of the items that make up the RAN

\begin{tabular}{|c|c|c|c|c|c|c|c|c|}
\hline & 4 years & 5 years & 4 years & 5 years & 4 years & 5 years & 4 years & 5 years \\
\hline $\begin{array}{c}\text { RAN } \\
\text { numbers }\end{array}$ & 2.316 & 1.120 & .827 & .131 & -.512 & 1.217 & -1.572 & -.296 \\
\hline $\begin{array}{c}\text { RAN } \\
\text { letters }\end{array}$ & 2.739 & 1.227 & .602 & .262 & -2.045 & 1.112 & 2.578 & -.428 \\
\hline $\begin{array}{c}\text { RAN } \\
\text { colours }\end{array}$ & 1.854 & 1.503 & .535 & .182 & .788 & 1.262 & -.347 & -.248 \\
\hline $\begin{array}{c}\text { RAN } \\
\text { images }\end{array}$ & 1.873 & 1.496 & .482 & .196 & 1.016 & 1.300 & .361 & -.207 \\
\hline
\end{tabular}

Then, after the development of Kaiser-Meyer-Olkin test and Bartlett sphericity test with satisfactory results determining the adequacy of samples (4 years: $\mathrm{KMO}=.66 \mathrm{y} \chi^{2} 21=138.31, \mathrm{p}<.001 ; 5$ years: $\mathrm{KMO}=.69$ y $\left.\chi^{2} 21=231.75, \mathrm{p}<.001\right)$, we proceeded to carry out an exploratory factor analysis in the subsample of 4 years children, and another in the subsample of 5 years children. Thus, to determine the structure of the instrument, an EFA was performed with direct oblimin rotation to analyze the variance the different items that make up the test share.

As shown in Table 2, in the subsample 1 of the 4 years old participants $(n=100)$, it has been possible to highlight a two-factor model that can explain the $70.35 \%$ of the total variance. The first factor includes the components of colors and images and explains the $37.44 \%$ of the variance, while the factor 2 includes the components of numbers and letters and explains the $32.91 \%$ of the variance.

Looking from a theoretical point of view the variables in each factor, it has been possible to observe that RAN components in the first factor have less to do with the education of children and, above all, that don't have to do with an arbitrary attribution of the label to the stimulus. Moreover, in the factor two is possible to find the components of "numbers" and "letters", whose name is not associated directly to the morphology of the stimulus, but requires a grapheme-phoneme conversion process which is more arbitrary and characteristic of a more advanced level of education and of reading-writing development. So, taking as a reference the discrimination showed by De Jong in his work of 2011, we decided to name the factor 1 "non-alphanumeric components" and factor 2 "alphanumeric components".

Table 2. EFA configuration matrix in 4 years old children

\begin{tabular}{ccc}
\hline & Factor 1 & Factor 2 \\
\hline RAN numbers & .022 & $\mathbf{. 7 6 9}$ \\
RAN letters & .026 & $\mathbf{. 7 7 8}$ \\
RAN colours & $\mathbf{. 7 0 4}$ & .061 \\
RAN images & $\mathbf{. 7 9 8}$ & .238 \\
\% explained variance & 37.44 & 32.91 \\
\hline
\end{tabular}


A similar pattern is found in the subsample 1 of 5 years old children $(n=100)$, where both factors explained $59.99 \%$ of the total variance (see Table 3). Again, the factor 1 gathers the components of "colors" and "images" and explains the $36.37 \%$ of the variance, while factor 2 components are "numbers" and "letters" explaining $23.62 \%$ of the variance.

However, it seems important to note that, with respect to the pattern obtained from the sample of children aged 4 years, this model not only explains a smaller percentage of the total variance, but also the factor loadings of the different components are lower, indicating a less force of differentiation of the two factors found.

Table 3. EFA configuration matrix in 5 years old children

\begin{tabular}{ccc}
\hline & Factor 1 & Factor 2 \\
\hline RAN numbers & .416 & $\mathbf{. 5 6 8}$ \\
RAN letters & .315 & $\mathbf{. 6 7 0}$ \\
RAN colours & $\mathbf{. 4 4 1}$ & .294 \\
RAN images & $\mathbf{. 6 1 0}$ & .355 \\
\% explained variance & 36.37 & 23.62 \\
\hline
\end{tabular}

In order to confirm the models obtained in the exploratory factor analysis it was conducted a confirmatory factor analysis (CFA). As shown in Table 4, the model tested with the subsample 2 of children of 4 years $(n=119)$ has a very good fit, since the absolute index Satorra-Bentler Chi-square is 118. 788 with 6 degrees freedom; the parsimony RMSEA index is .001 ( $<.05$ good fit, between .05 and .08 acceptable, $>.08$ mediocre; Browne \& Cudeck, 1993) and the comparative index CFI corresponds to 1 (.90-.95 acceptable, >.95 good; Hu \& Bentler, 1999).

Table 4. Goodness of fit indexes of the CFA model in 4 years old children

\begin{tabular}{ccccc}
\hline$\chi^{2}$ s-B & df & $\chi^{2}$ s-B/gl & RMSEA & CFI \\
\hline 118.788 & 6 & 19.7 & .001 & 1 \\
\hline
\end{tabular}

Figure 1 shows the drawing of the CFA model developed with the subsample 2 (children of 4 years). As shown in the path diagram, standardized model weights ranged from .47 (RAN numbers) and .83 (RAN colors) while the covariance between the two factors was found to be 0.62 .

Figure 1. Path diagram of the CFA model in 4 years old children

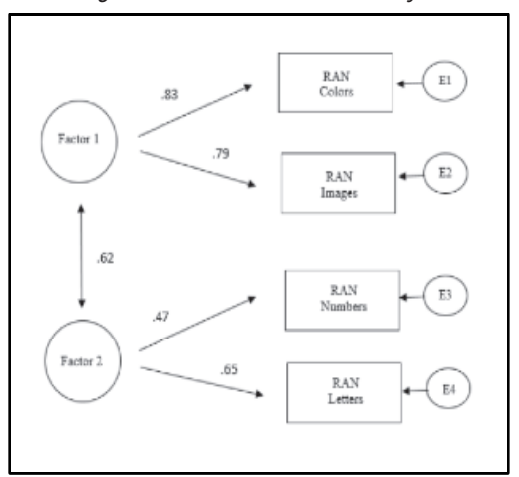


The same procedure was developed with the subsample 2 of children of 5 years $(n=123)$. It was carried out a CFA to confirm the pattern obtained from the exploratory analysis. As shown in Table 5, the Satorra-Bentler Chi Square for this model is 257.653 with 12 degrees of freedom, so the ratio is 21.47. Likewise, the goodness of fit indexes are located on the edge of acceptability being RMSEA .08 (<.05 good fit, between .05 and .08 acceptable, >.08 mediocre; Browne \& Cudeck, 1993) and the CFI .85 (.90-.95 acceptable, >.95 good; Hu \& Bentler, 1999). These values indicate and reconfirm a lower robustness of the model in the sample of 5 years old children in comparison with the one obtained with younger children.

Table 5. Goodness of fit indexes of the CFA model in 5 years old children

\begin{tabular}{ccccc}
\hline$\chi_{\text {S-B }}^{2}$ & df & $\chi^{2}$ S-B/df & RMSEA & CFI \\
\hline 257.653 & 12 & 21.47 & .08 & .85 \\
\hline
\end{tabular}

In addition, Figure 2, which shows a drawing of this model, highlights that the standardized weights ranged from .27 to.56, while the covariance between the two factors was .83. This latter setting allows deducing a great similarity between the two factors of the RAN confirming once again less discriminative power of the model and closer to the 4 components of the test.

Figure 2. Path diagram of the CFA model in 5 years old children

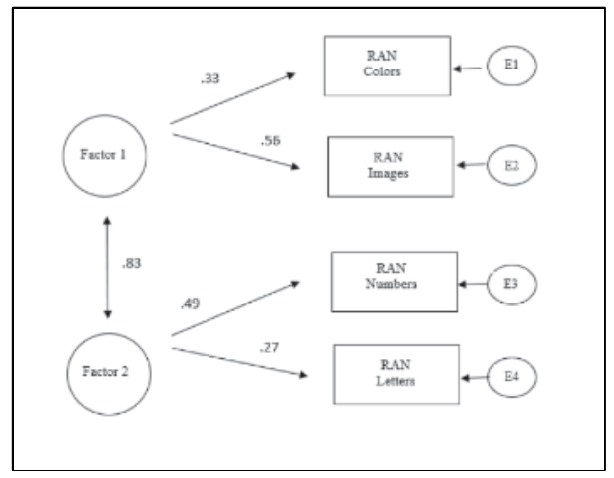

The next objective was to determine the reliability, validity and internal consistency of the test. So, we developed an analysis of the composite reliability IFC (Fornell \& Larcker, 1981), of the average extracted variance AVE (Fornell \& Larcker, 1981) and of the Cronbach (1951) for each factor confirmed with the CFA, both for the 4 years old children sample and the 5 years old one.

As shown in Table 6, the model obtained in younger participants present in both factors a very good Cronbach's alpha (following Nunnally \& Bernstein classification developed in 1994 for whom values higher than .70 are considered acceptable), without exceeding the threshold of .90 that, as referred O'Rourke, Hatcher \& Stepanski in his 2005 work, indicate the existence of redundant items on the scale. Also, in both factors it has been possible to highlight a satisfactory average extracted variance (AVE), since all the values over 0.5 are considered acceptable (Fornell \& Larcker, 1981). With respect to the reliability composed index IFC whose interpretation is similar to the one used for Cronbach's alpha, it can be said that the factor 2 has a very satisfactory value being of .830 , while the factor 1 is considered reasonably good (.673). 
Table 6. Reliability indexes in the 4 years old children simple.

\begin{tabular}{cccc}
\hline & IFC & AVE & Cronbach's $\boldsymbol{\alpha}$ \\
\hline $\begin{array}{c}\text { Factor 1 } \\
\text { "non-alphanumeric } \\
\text { components" }\end{array}$ & .673 & .580 & .81 \\
$\begin{array}{c}\text { Factor 2 } \\
\text { "alphanumeric } \\
\text { components" }\end{array}$ & .830 & .592 & .84 \\
\hline
\end{tabular}

Table 7 shows the indices of reliability and validity of the test found in the AFC model for 5 years old children. As it's possible to see, the indexes in this sample, despite being acceptable according to the criteria described above, are not as good as those featured in the model of the younger participants. It seems important to underline especially Cronbach's alpha values for the factor "nonalphanumeric components" being .89 and .90 in factor 2. Despite being a very satisfactory alpha, in this test validation context, its proximity to the value of .90 suggests the existence in the same test of items very close to each other, which would undermine the test's ability of discrimination.

Table 7. Reliability indexes in the 5 years old children sample.

\begin{tabular}{lccc}
\hline & IFC & AVE & $\begin{array}{c}\text { Cronbach's } \\
\boldsymbol{\alpha}\end{array}$ \\
\hline $\begin{array}{l}\text { Factor 1"non-alphanumeric } \\
\text { components" }\end{array}$ & .632 & .512 & .89 \\
$\begin{array}{l}\text { Factor 2 "alphanumeric } \\
\text { components" }\end{array}$ & .694 & .498 & .90 \\
\hline
\end{tabular}

\section{DISCUSSION}

Even if cognitive aspects related to reading are well known, there are still few studies that investigate how these variables develop in the early years of schooling. Through the study of these components, an effective analysis of reading even before reaching a full and complete acquisition of it can be addressed (Norton \& Wolf, 2011). Therefore, understanding the development and function of variables such as speed of naming, defined as the child's ability to recognize and name familiar stimuli quickly, is needed. However, this analysis has been hampered by the absence of an unique, reliable and standardized instrument that allow the sharing and comparison of the results found in different studies (Araújo, Petersson, Reis \& Faísca, 2014).

To meet this need, our research has focused on the standardization and factorization of the RAN test developed by Wolf and Denkla in 2003. Our analyses have highlighted a composite of two factors that collect the 4 components that characterize the test structure. A first factor collects the components that can be considered more intuitive and directly associated with the morphology of the stimuli: "RAN colors" and "RAN images". The second factor, meanwhile, collects components related to an arbitrary attribution of the label to the item and involve a more advanced level of schooling and of reading-writing development, that is, "RAN letters" and "RAN numbers". Therefore, and following the differentiation shown by De Jong in a study in 2011, it was decided to name the two factors respectively as "non-alphanumeric components" and "Alpha-numeric components ".

Moreover, thanks to the application of the test to 4 and 5 years old children and an analysis of 
its structure at both ages, it has been possible to study the development of the speed of naming over the first two years of the beginning of children literacy.

The results of this study allow highlighting a gradual shift of weight that each component of the test has in the two analyzed years and a consequent reduction in the discrimination ability of the test. Indeed, the most intuitive components as RAN colors and RAN images appear to be much more accessible than arbitrary ones (RAN numbers and RAN letters) for 4 years old children while this differentiation becomes weaker at 5 years. Those claims are confirmed by observing the mean scores obtained by the children in the different components in both age levels. Indeed, in 4 years old children the first factor's components ("non-alphanumeric components") are associated with better scores than the second ones ("alphanumeric components"), indicating, in these children, greater ease and speed in recognizing and naming more basic stimuli, such as colors and images. However, scores for 5 years old children not only improve on the 4 components (indicating a progressive improvement of the overall process) but become more homogeneous between them, suggesting a decrease in the facilitator power of colors and images found the year before.

These statements found their confirmation in the exploratory analyzes developed. The factor loadings of the components of the model with 5 years old participants are smaller, indicating a minor difference between the two factors found. Furthermore, the model obtained in older children explained only $59.99 \%$ of the variance, while factors in four years participants are able to explain the $70.35 \%$ of the total variance. Finally, the AFC has allowed us to confirm that the pattern obtained from the sample of five years children presents a worse fit, being all indexes on the edge of acceptability. Also, this model has found a covariance between the two factors of .83, suggesting again a smaller capacity of discrimination of the model and a greater similarity at this age between the 4 components of the test. This similarity is confirmed by the Cronbach's alpha analyzed to assess the test's reliability. Indeed the two factors model for 5 years old children present a very high alpha (.89 and .90) which, as referred 0'Rourke, Hatcher \& Stepanski in their 2005 work, suggests the existence of redundant items.

These results are in line with previous studies that emphasize that scores in the RAN in more basic stimuli such as objects and colors can be considered at early age as good predictors of a later reading development (Kirby at al., 2003; Landerl \& Wimmer, 2008); whereas when the reading-writing development of children and their education have already begun there is an increasingly importance of the more arbitrary components of numbers and colors (De Jong, 2011). These findings also confirm those obtained in studies that our research group is developing and highlighting a gradual reduction in the difference in the weight of the 4 components of the RAN.

In conclusion, this study not only allows to analyze the structure of the instrument "RAN" and confirm its statistical validity and reliability, but also describes how one of the cognitive functions that literature more relates to reading-writing development, as the speed of naming is, works over the early years of schooling.

\section{REFERENCES}

Araújo, S., Inácio, F., Francisco, A., Faísca, L., Petersson, K. M., \& Reis, A. (2011). Component processes subserving rapid automatized naming in dyslexic and non-dyslexic readers. Dyslexia, 17, 242-255. doi:10.1002/dys.433.

Araújo, S., Reis, A., Petersson, K. M., \& Faísca, L. (2014, October 20). Rapid Automatized Naming and Reading Performance: A Meta-Analysis. Journal of Educational Psychology. Advance online publication. http://dx.doi.org/10.1037/edu0000006.

Babayig it, S., \& Stainthorp, R. (2010). Component processes of early reading, spelling, and narra- 
tive writing skills in Turkish: A longitudinal study. Reading and Writing, 23, 539-568. doi:10.1007/s11145-009-9173-y.

Bowers, P. G., \& Wolf, M. (1993). Theoretical links among naming speed, precise timing mechanisms and orthographic skill in dyslexia. Reading and Writing, 5, 69-85. doi:10.1007/BF01026919.

Chiappe, P., Stringer, R., Siegel, L. S., \& Stanovich, K. E. (2002). Why the timing deficit hypothesis does not explain reading disability in adults. Reading and Writing, 15, 73-107. doi:10.1023/A:1013868304361.

Clarke, P., Hulme, C., \& Snowling, M. (2005). Individual differences in RAN and reading: A response timing analysis. Journal of Research in Reading, 28, 73-86. doi:10.1111/j.14679817.2005.00255.x.

Cobbold, S., Passenger, T., \& Terrel, C. (2003). Serial naming speed and the component elements of speech time and pause time: Relationships with the development of word-level reading in children aged four to five years. Journal of Research in Reading, 26, 165-176. doi:10.1111/14679817.00194

Cunningham, A. E. (2006). Accounting for children's orthographic learning while reading text: Do children self-teach? Journal of Experimental Child Psychology, 95, 56-77.

Cutting, L. E., \& Denckla, M. B. (2001). The relationship of serial rapid naming and word reading in normally developing readers: An exploratory model. Reading and Writing, 14, 673-705. doi:10.1023/A:1012047622541.

Georgiou, G. K., Parrila, R., Kirby, J. R., \& Stephenson, K. (2008). Rapid naming components and their relationship with phonological awareness, orthographic knowledge, speed of processing, and different reading outcomes. Scientific Studies of Reading, 12, 325-350. doi:10.1080/10888430802378518.

Kirby, J. R., Parrila, R. K., \& Pfeiffer, S. L. (2003). Naming speed and phonological awareness as predictors of reading development. Journal of Educational Psychology, 95, 453-464. doi:10.1037/0022-0663.95.3.453.

Kirby, J. R., Roth, L., Desrochers, A., \& Lai, S. (2008). Longitudinal predictors of word reading development. Canadian Psychology, 49,103-110. doi:10.1037/0708-5591.49.2.103.

Landerl, K., \& Wimmer, H. (2008). Development of word reading fluency and spelling in a consistent orthography: An 8-year follow-up. Journal of Educational Psychology, 100, 150-161. doi:10.1037/0022-0663.100.1.150.

Moll, K., Fussenegger, B., Willburger, E., \& Landerl, K. (2009). RAN is not a measure of orthographic processing: Evidence from the asymmetric German orthography. Scientific Studies of Reading, 13, 1-25. doi:10.1080/10888430802631684.

Norton, E. S., \& Wolf, M. (2011). Rapid automatized naming (RAN) and reading fluency: Implications for understanding and treatment of reading disabilities. Annual Review of Psychology, 63, 427-452.

Papadopoulos, T. C., Georgiou, G. K., \& Kendeou, P. (2009). Investigating the double-deficit hypothesis in Greek. Journal of Learning Disabilities, 42, 528-547. doi:10.1177/0022219409338745.

Pennington, B. F., Cardoso-Martins, C., Green, P. A., \& Lefly, D. L. (2001). Comparing the phonological and double deficit hypotheses for developmental dyslexia. Reading and Writing, 14, 707-755. doi:10.1023/A:1012239018038.

Roman, A. A., Kirby, J. R., Parrila, R. K., Wade-Woolley, L., \& Deacon, S. H. (2009). Toward a comprehensive view of the skills involved in word reading in grades 4,6 , and 8 . Journal of Experimental Child Psychology, 102, 96-113. doi:10.1016/j.jecp.2008.01.004. 
Scarborough, H. S. (1998). Predicting the future achievement of second graders with reading disabilities: Contributions of phonemic awareness, verbal memory, rapid naming, and IQ. Annals of Dyslexia, 48, 115-136. doi:10.1007/s11881-998-0006-5.

Schatschneider, C., Carlson, C. D., Francis, D. J., Foorman, B. R., \& Fletcher, J. M. (2002). Relationship of rapid automatized naming and phonological awareness in early reading development: Implications for the double-deficit hypothesis. Journal of Learning Disabilities, 35, 245-256. doi:10.1177/002221940203500306.

Vaessen, A., Bertrand, D., Tóth, D., Csépe, V., Faísca, L., Reis, A., \& Blomert, L. (2010). Cognitive development of fluent word reading does not qualitatively differ between transparent and opaque orthographies. Journal of Educational Psychology, 102, 827- 842. doi:10.1037/a0019465.

Wolf, M., \& Bowers, P. G. (1999). The double-deficit hypothesis for the developmental dyslexias. Journal of Educational Psychology, 91, 415-438. doi:10.1037/0022-0663.91.3.415.

Wolf, M., Bowers, P. G., \& Biddle, K. (2000). Naming-speed processes, timing, and reading: A conceptual review. Journal of Learning Disabilities, 33, 387-407. doi:10.1177/002221940003300409.

Wolf, M., O'Rourke, A. G., Gidney, C., Lovett, M., Cirino, P., \& Morris, R. (2002). The second deficit: An investigation of the independence of phonological and naming speed deficits in developmental dyslexia. Reading and Writing, 15, 43-72. doi:10.1023/A:1013816320290 